\title{
Bandwidth and Radiation Management of GSM System using Fuzzy Logic
}

\author{
Bhargav V. Gokalgandhi \\ Department of EXTC \\ D. J. Sanghvi College \\ Mumbai.
}

\author{
Dereyk D'souza \\ Department of EXTC \\ D. J. Sanghvi College \\ Mumbai.
}

\author{
Arun J. Varia \\ Department of EXTC \\ D. J. Sanghvi College \\ Mumbai.
}

\author{
Sagar Samant \\ Department of EXTC \\ D. J. Sanghvi College \\ Mumbai.
}

\begin{abstract}
Bandwidth and the radiation pattern of an antenna play a crucial role in long as well as short distance communication. Nowadays, the increasing population and the unequal use of communication channels are making it difficult to manage the bandwidth of a communication system. Also, the radiation of the antenna is inefficiently used in many areas. One of the solutions to these problems is using fuzzy logic. It will help in easier management of the database. The allocation of bandwidth and the control of radiation pattern will become extremely simple. All the factors will be automatically updated according to the existing requirements thus, making the system a self-learning system.
\end{abstract}

\section{Keywords}

Fuzzy, Bandwidth, Radiation, Directivity, Inference, fuzzification, defuzzification.

\section{INTRODUCTION}

Since the advent of the communication age, antennas have become an integral part of development of any type of communication system. Any system, whether used for communicating long or short distances, has to use specific types of antennas. Hence, the parameters of antennas have a substantial effect on the designing of systems. Some parameters such as the bandwidth and the effective radiation have a great influence on the efficient working of the system. Hence, these parameters have to be used as effectively as possible.

But due to the ever increasing demands of the ever increasing population of the world, there is a lot of strain on the existing resources. This strain is especially on the infrastructure of the developing countries. Hence, something has to be done to use the existing resources as efficiently as possible. Here, as there is unequal distribution of population in developing countries, there are a lot of problems in setting up areas where the bandwidth of the antennas can be effectively used. In many urban areas the number of users using these communication devices is much more than in the rural areas. Hence, the bandwidth of the antenna is severely affected and also a lot of radiation power has to be maintained whilst, in rural areas the number of users is comparatively less and hence larger bandwidth is not required.

Fuzzy logic is basically a mathematical logic wherein the true value of any event can be assumed in the continuum of values between 0 and 1 . As the name suggests, it is a method of reasoning that is vague or fuzzy. By vague it means that there are multiple possible values that can represent a given scenario. It can be like evaluating a statement and defining a variable to it as partly true, completely true or completely false. We can say that fuzzy logic is a method in which a given event is justified in various degrees of trueness. As it is seen that the variable is defined a non-numeric value rather that a numerical one in fuzzy logic. Fuzzy logic is governed by a predefined set of rules. It is a tool to deal with uncertain events that are too complex to deal with normal methods. These approximations can be really useful when dealing with such complex situations where true or exact values tend to be hindering.

Here, Fuzzy Logic is being used to control or to set the radiation pattern and the bandwidth of an antenna or a base station in such a way that maximum radiation is utilized and there is minimum wastage of bandwidth. This will make the base stations more flexible in terms of frequency utilization and more efficacious in terms of radiation.

\section{PROJECT DESCRIPTION}

In a GSM Network, the multiplexing is done in two ways. It is TDM [Time Division Multiplexing] and FDM [Frequency Division Multiplexing]. This means that the channels in GSM are divided in frequency as well as time. This is done with a sole purpose of efficiently using the bandwidth provided.

But, at the same time, the bandwidth required by each area and the amount of time slots required in each channel can vary depending on the magnitude of the users in that area. If supposedly the number of users in an area A is less and still the bandwidth provided to them is same as that of the bandwidth provided to more number of users in some other area $\mathrm{B}$, then the bandwidth in area A is unused while that in area B is strained as the channel capacity in area $\mathrm{B}$ will be lower than the required information rate.

If a particular base station region is such that there are irregularities in the use of its radiation. It means that in some areas of that cell there is a lot of use of the radiation as the number of users is high or the radiation cannot penetrate to longer distances because of losses due to obstacles, then the radiation pattern or the radiation power has to be changed accordingly so that maximum radiation can be used with minimum power wastage.

Now, all the above examples show that controlling the bandwidth and the radiation is very important for efficient communication in areas where the population density is high and its distribution is unequal. These problems mentioned above can be easily controlled and a system can be designed using fuzzy logic. 


\section{OUTLINE FOR FUZZIFICATION}

Here, fuzzy logic is being used to set the base values of the bandwidth and the amount of radiation power of a region. Now, the bandwidth of a given base station is decided according to the number of users present in that base station. If a single base station is considered and the scenario happens, as mentioned above, i.e. bandwidth inequality between areas $\mathrm{A}$ and $\mathrm{B}$, then in such case the values or the IF-THEN logic will be set in such a way that the area in which there is more usage or requirement of bandwidth will receive more bandwidth and the area in which less number of users are present will receive lesser bandwidth.

Another part is the radiation power part. In this part the radiation power or the directivity of the antenna of the base station is maintained according to the requirement of that region. The requirements are the number of users, the distance with respect to the base station, difference between the density of users in different areas of the base station, etc.. According to these specifications the values are set and manipulated. One can observe that rigid boundaries are not to be maintained and the values can change according to the base station requirements.

The logic here is developed using two very basic inputs i.e. the amount of calls in a stipulated time period and the distance of the users from a given base station. The amount of calls of one region will be compared with the amount of calls in another region of the same base station and the bandwidth allocation will change accordingly. Also, the amount of data usage of one base station is compared with the amount of data usage of other base stations and the bandwidth assigned to each base station can be changed accordingly.

For the radiation power density, the distance, the mobility and also the data usage of the users of a given area are taken as the input and the output is the change in the radiation power and change in the directivity of the antenna. Mobile antennas, which are usually Omnis-directional increase their directivity towards a certain region. Or if the antenna is directed in a certain region, it becomes les directional and the power density in all regions starts becoming equal.

The mobility of the users will change the antenna radiation patterns only when there are a large number of users moving from one location to another with very high speed. But, for our experiment we are considering the movement of very less number of users from one location to another and so the movement is not considered as an input.

\section{INFERENCE}

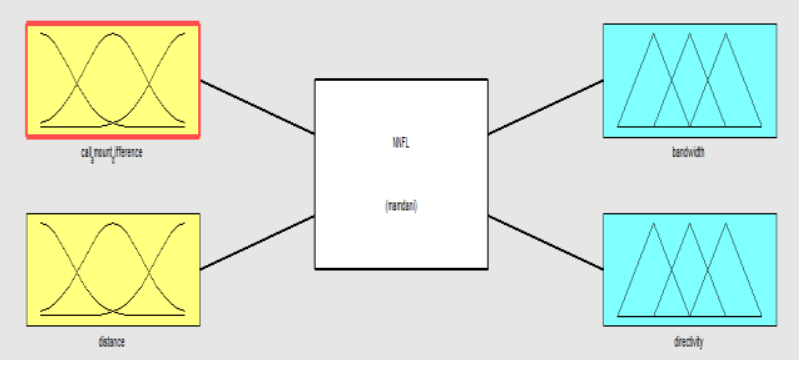

Fig. 1 Basic Fuzzy system with inputs and outputs

The inputs entered in the fuzzy logic inference system are the difference of amount of calls in a given area and the comparative distance of the users in a given area. Only a single base station is considered. Multi base station logic is not implemented in this system. The outputs were the change in the bandwidth and the change in the directivity of the antenna.
The IF-THEN logic or the rules of the fuzzy system and all the definitions of the system are with respect to Mamdani logic. The input patterns are as shown below:

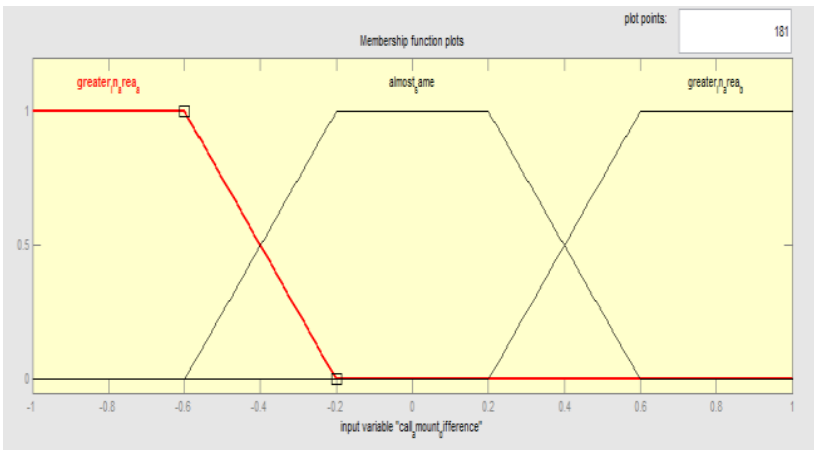

Fig. 2 Difference in amount of calls in area a and area b

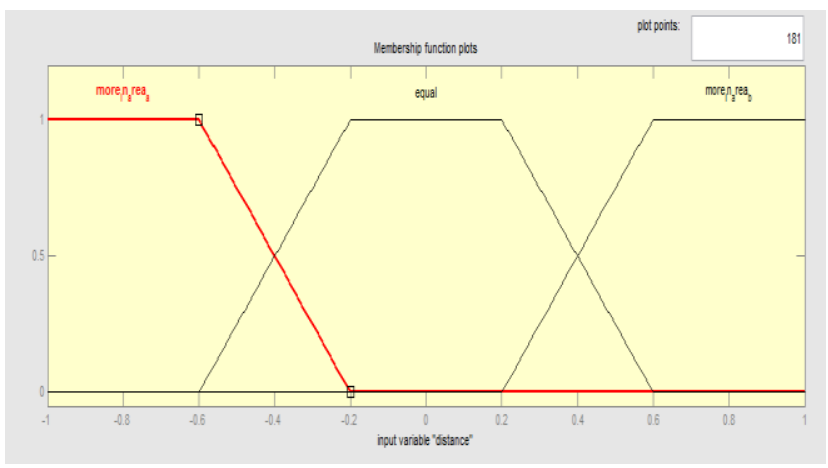

Fig. 3 Distance of users from the base station

The above two figures show the two inputs. The first input shows the amount of calls in a stipulated time period in the two areas. It doesn't actually show the amount of calls. It shows the comparative amount or the difference in the number of calls happened in each area. The second figure shows the comparative distance of the users, present in both the areas, from the base station.

Here, negative values in both the figures indicate that the amount of calls and the distance of the users from the base station is more in 'area a' whereas positive values show the same for users in 'area b'. As the values tend toward zero, this shows that there is not much difference in the comparative distance and the amount of calls generated which will not affect the bandwidth and the radiation pattern on a larger scale.

The output patterns are as shown below:

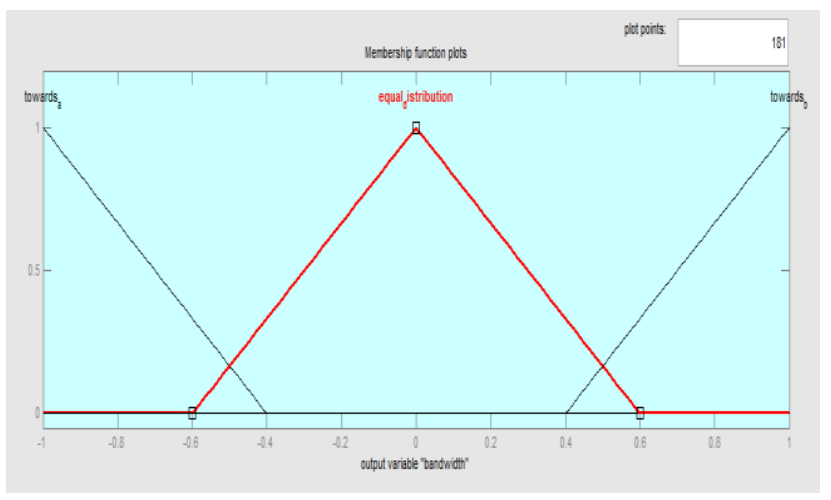

Fig. 4 Change in Bandwidth 


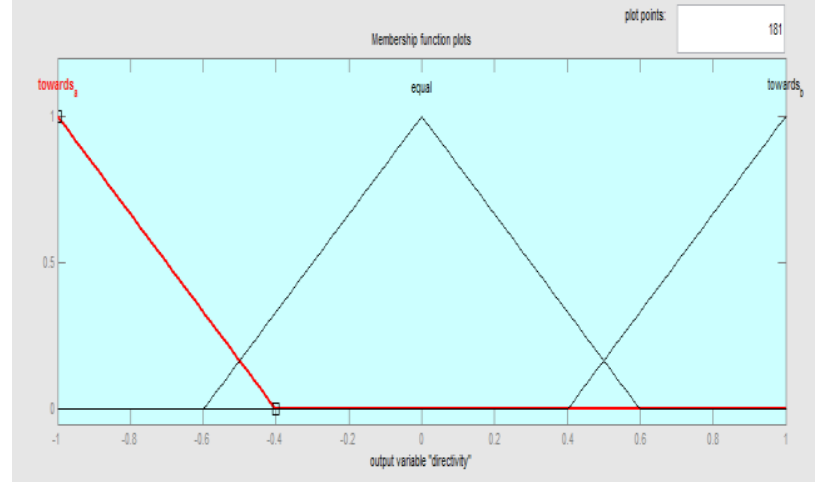

Fig, 5 Change in Directivity or radiation pattern

The above figures show the output patterns and values which are to be considered according to the inputs given. Here too, the negative values i.e. the values on the left side of the graph show that the outputs are tending towards 'area a' and the positive values on the right side for 'area b'. The centre values which are the values near to zero show that there is no major change in the output parameters. This means that the center values will indicate that there is no major difference between the input values of 'area a' and 'area b'.

The rules are defined as follows:

4.1.1 IF call amount is greater in a and distance is greater in a THEN bandwidth is towards a and directivity is towards a.

4.1.2 IF call amount is greater in a and distance is equal THEN bandwidth is towards $a$ and directivity is equal.

4.1.3 IF call amount is greater in a and distance is greater in $b$ THEN bandwidth is towards $a$ and directivity is towards $b$.

4.1.4 IF call amount is same and distance is greater in a THEN bandwidth is equal and directivity is towards a.

4.1.5 IF call amount is same and distance is equal THEN bandwidth is equal and directivity equal.

4.1.6 IF call amount is same and distance is greater in $b$ THEN bandwidth is equal and directivity is towards $b$.

4.1.7 IF call amount is greater in $b$ and distance is greater in a THEN bandwidth is towards $b$ and directivity is towards $a$.

4.1.8 IF call amount is greater in $b$ and distance is equal THEN bandwidth is towards $b$ and directivity is equal.

4.1.9 IF call amount is greater in $b$ and distance is greater in $b$ THEN bandwidth is towards $b$ and directivity is towards $b$.

In the above defined rules, the call amount and the distance mentioned are comparative quantities. If it is stated that amount of call in 'area a' is greater, then it means it is greater than the amount of calls generated in 'area b'. Same applies for the distance factor.

\section{DEFUZZIFICATION}

After the inputs, outputs and the rules are specified, the result obtained will be through the defuzzification process. The defuzzification process uses the centroid method. In this method, the centroid values of the resultant figure obtained from the given values are multiplied with the area of the figure and the resultant is divided by the total area. This value is the final value obtained for a given condition. In the same way, for other set of rules or conditions the defuzzified value will be different.
These defuzzified values will be used by the system to define and manipulate its bandwidth and radiation pattern. Following figure shows an example of the defuzzified value.

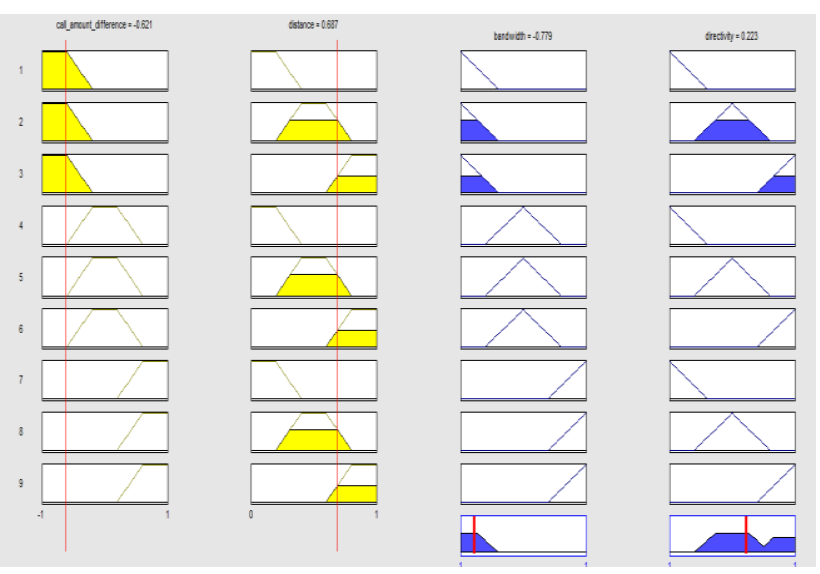

Fig. 6 Defuzzified value for one of the IF-THEN conditions

The above figure shows the defuzzified values of bandwidth and directivity for the condition that the amount of calls is more in 'area a' and the comparative distance of the users is greater in 'area b'. The value of bandwidth is -0.779 which shows that the bandwidth will increase for users in 'area a' and decrease for users in 'area b'.

In the same way, the value of directivity comes out to be 0.233 . Now, this value is positive. This means that the directivity of the antenna should be towards 'area b'. But one should note that the value is less and is nearer to 0 . Hence, one cannot just change the radiation parameters to the extreme. The change of the directivity will be of a very low magnitude.

The table for different defuzzified values is shown below:

Defuzzified Values for all the Rules

\begin{tabular}{|c|c|l|l|}
\hline \multicolumn{2}{|c|}{ Input values } & \multicolumn{2}{c|}{ Output values } \\
\hline $\begin{array}{c}\text { Difference } \\
\text { in the } \\
\text { number of } \\
\text { calls or data } \\
\text { usage }\end{array}$ & $\begin{array}{c}\text { Camparative } \\
\text { distance }\end{array}$ & Bandwidth & Directivity \\
\hline-0.759 & -0.877 & -0.807 & -0.807 \\
\hline-0.759 & 0.00985 & -0.807 & $-1.16^{*} 10^{\wedge}-19$ \\
\hline-0.759 & 0.522 & -0.807 & 0.466 \\
\hline 0.0197 & -0.857 & $-1.16^{*} 10^{\wedge}-$ & -0.807 \\
\hline 0.0197 & -0.325 & $-1.16^{*} 10^{\wedge}-$ & -0.164 \\
\hline 0.0197 & 0.936 & $-1.16^{*} 10^{\wedge}-$ & 0.807 \\
\hline 0.571 & -0.591 & 0.639 & -0.745 \\
\hline 0.571 & 0.0887 & 0.639 & $-1.16^{*} 10^{\wedge}-19$ \\
\hline 0.571 & 0.591 & 0.639 & 0.745 \\
\hline
\end{tabular}


The above table shows the different defuzzified values of bandwidth and directivity with respect to the two inputs. We have discussed one example before the table to illustrate the way in which the defuzzified values are being defined.

In the table, we are defining all the defuzzified values for all the different rules that we have assigned to the fuzzy inference system. We have varied the two inputs according to the rules and then noted the different defuzzified values for bandwidth and directivity. We have kept the first input i.e. the difference in the amount of calls as constant first and varied the comparative distance. Now, the defuzzified values show that the Bandwidth values are only proportionally varying with respect to the first input and the directivity is only dependent on the distance factor. Further discussion on that will be done afterwards by using the graphs.

Now, from the above table, it can be seen that the first input values, when they are negative, are taken to a large magnitude toward the negative side and hence the bandwidth is seen to have a negative value of the same proportion. As, the values of the data usage are tending to zero, the bandwidth value becomes very small i.e. it becomes negligible. This means that the bandwidth distribution will be same in both the areas. Then, as the input becomes positive, the bandwidth becomes positive. But, here the input magnitude is small. This will result in very less change in the amount of bandwidth.

In the same way, the value of directivity of antenna also changes. It is observed that we have taken more variation in the values of directivity, but the end result is the same as of that in bandwidth. The change in directivity or radiation pattern as shown in the fifth row tells us that when the comparative distance is not of a great magnitude, then the change in directivity is also very less.

Overall, it is seen that only for values around -0.2 to -0.6 and 0.2 to 0.6 , there is major change in the bandwidth and the directivity or the radiation pattern when the inputs are in these regions.

\section{CONCLUSION}

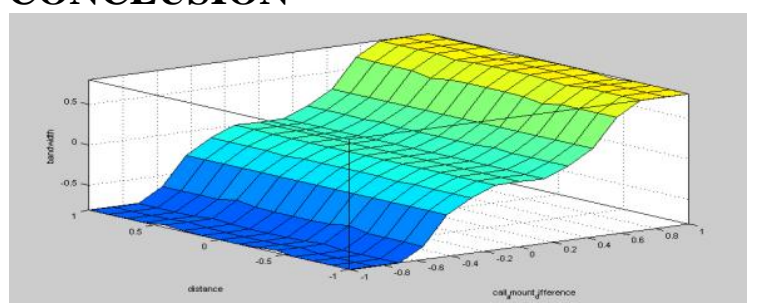

Fig. 7 Graph of Bandwidth vs. Distance and Amount of calls

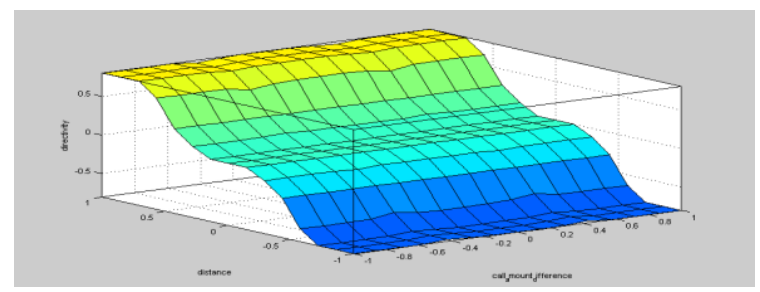

Fig. 8 Graph of Directivity vs. Distance and Amount of calls
The above figures show the final input- output relations after Fuzzification and Defuzzification. These graphs show how the bandwidth and the directivity of the system varies with the variations in inputs.

The first figure shows the variation of bandwidth with the variations in the difference in the amount of calls or the data usage. It is seen that the bandwidth is totally unaffected by the variations in the distance. It varies only with the amount of use of data. The second figure shows the variations in the directivity where it is seen that directivity varies only according to the variations in distance and it is independent from variations in data usage or calls.

Thus, it is seen that GSM system can be easily managed, controlled and its efficiency can be increased using fuzzy logic We have seen that the system becomes extremely flexible by just using fuzzy logic for two of its factors. So, if fuzzy logic is used for all the factors of the system or even the factors that have a major effect on the system, then the system becomes a autolearning system and it becomes very easy to manage it which directly and indirectly saves a lot of time and cost. Also, by introducing neural networks, one can easily make the system which can adapt to any given situation.

Lastly, factors like the movement of the users, the importance of the information or the security of the information which is being carried around is not being concerned with in this paper. If these factors are also considered then it will make the system more efficient, flexible and secure.

\section{ACKNOWLEDGMENT}

We are highly thankful to Prof. Pradeep Yadav and Prof. Kelkar timely and valuable suggestions given by then throughout the completion of this paper. We would like to express our special gratitude and thanks to them for giving us such attention and time. We are also thankful to the EXTC department of D. J. Sanghvi College of Engineering for giving us this opportunity to put forth our ideas and to make our creative utopias practical. Our thanks and appreciations also go to people who have willingly helped us out with their abilities.

\section{REFERENCES}

[1] S. N. Shivanandan and S. N. Deepa, Principles of Soft Computing. $2^{\text {nd }}$ Edition, Wiley.

[2] Theodore S. Rappaport, Wireless Communications: Principles and Practice, $2^{\text {nd }}$ Edition, Pearson.

[3] Andreas Pitsillides, Ahmet Sekercioglu, "Fuzzy logic based Congestion control".

[4] ALI HAKAN IȘIK, ERKAN AFACAN, "Control of Cell Planning with Fuzzy Logic in GSM System","Proceedings of the 8th WSEAS International Conference on Telecommunications and Informatics".

[5] L. A. Zadeh. Fuzzy Logic. IEEE Computer. pp. 83-93, April 1988.

[6] Mathworks website for FIS webinar. 